\title{
An overview of Pneumocystis jirovecii pneumonia for the African generalist practitioner
}

\author{
I Govender ${ }^{1}$, OM Maphasha $^{1}$, S Rangiah ${ }^{2}$, C Steyn ${ }^{1}$
}

1. Department of Family Medicine, Sefako Makgatho Health Sciences University. P.O. BOX 222, MEDUNSA 0204. 2. Department of Family Medicine, University of Kwa Zulu Natal.

Emails:

Indiran.govender@gmail.com; olgamaphasha@yahoo.com; Rangiah@ukzn.ac.za; stencarien@yahoo.com

\begin{abstract}
Introduction: Pneumocystis jirovecii is the causative organism of Pneumocystis pneumonia (PCP) in humans, which is more common among immunocompromised patients. Classically patients present with fever, non-productive cough, and dyspnoea. In the HIV-infected individuals the symptoms may be subtle at first, but gradually progress over several weeks. In the HIV-uninfected patient, however, the duration of symptoms is shorter and more severe, mainly due to the increased inflammatory response of the HIV-uninfected patient.

Methods: This article focuses on the diagnostic methods and then the management and prophylaxis principles of PCP by reviewing the best current practices and guidelines in Africa.

Conclusion: This overview is presented by clinicians who have experience with PCP and is directed mainly at first-line healthcare providers.

Keywords: Pneumocystis jirovecii pneumonia, african generalist practitioner.

DOI: https://dx.doi.org/10.4314/ahs.v19i4.43

Cite as: Govender I, Maphasha OM, Rangiah S, Steyn C. An overview of Pneumocystis jirovecii pneumonia for the African generalist practitioner. Afri Health Sci.2019;19(4):3200-3207. https:/ / dx.doi.org/10.4314/ahs.v19i4.43
\end{abstract}

\section{Background, history and epidemiology of PCP}

The causative organism of Pneumocystis pneumonia (PCP) is the fungus Pneumocystis jirovecii. ${ }^{1}$ This organism was first classified as the protozoan Pneumocystis carinii, but the classification was changed in $1988 .^{2}$ This is the only fungus in the Pneumocystis group that causes disease in humans. ${ }^{3}$ Although the abbreviation PJP is sometimes used, PCP is still used for convenience to designate Pneumocystis pneumonia, and is the abbreviation for Pneumo Cystis (jirovecii) Pneumonia. ${ }^{4}$

This classification was based on the morphologic features and the resistance to classical antifungal agents. Both Carlos Chagas and Antonio Carini found Pneumocystis in

\section{Corresponding author: \\ I Govender, \\ Department of Family Medicine, \\ Sefako Makgatho Health Sciences University \\ P.O. BOX 222, MEDUNSA 0204.}

Email: indiran.govender@gmail.com the lungs of rats. ${ }^{2}$ Until the 1980s, the phylogenetic classification of Pneumocystis as a protozoan was based on the histological characteristics of its life cycle forms, trophic and cystic forms; inability to maintain in vitro cultures of the organism and successful response to treatment with anti-parasitic drugs, such as pentamidine or primaquine. ${ }^{5}$ The affinity of the species for the lung led to the identifier Pneumocystis. ${ }^{2}$ The microbe was first described in humans by Otto Jirovec, after whom the disease was subsequently been named.6 The full and correct classification of pneumocystis therefore took many years. ${ }^{2}$

PCP was first reported during World War II in crowded orphanages in Central and Eastern Europe among premature infants and malnourished children. ${ }^{3,7}$ In the 1980s, the incidence of PCP increased dramatically with the emergence of HIV. However, the incidence of the disease in the HIV-infected population has decreased significantly due to the use of anti-retroviral agents and PCP prophylaxis. An interesting finding in Sweden during the last stage of a study that was done between 1991 and 2001 showed that $75 \%$ of PCP patients were HIV-uninfected. This is the result of other immunosuppressive conditions and treatments. ${ }^{3}$

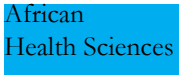

3200
C) 2019 Govender et al. Licensee African Health Sciences. This is an Open Access article distributed under the terms of the Creative commons Attribution License (https://creativecommons.org/licenses/BY/4.0), which permits unrestricted use, distribution, and reproduction in any medium, provided the original work is properly cited. 
Pneumocystis organisms have been described in most mammals. However, the organisms are host specific, and those that are infectious to humans will not infect any other mammalian species. ${ }^{2}$ Pneumocystis jirovecii is distributed worldwide and PCP has been described in all continents except Antarctica. ${ }^{8}$

PCP is one of the most common potentially life-threatening opportunistic diseases amongst patients with HIV/ AIDS, especially in those with CD4 cell counts of less than 200 cells $/ \mathrm{mm}^{3.2,9,10,11}$ It is classified as an AIDS-defining illness and places the patient in the World Health Organization clinical stage s.1,13 $^{4}$ Other factors associated with the risks for PCP infection in the HIV-infected population include oral thrush, recurrent bacterial pneumonia, previous PCP infection, unintentional weight loss and high viral load.13 Even though PCP remains an important cause of HIV-associated pneumonia, the incidence of PCP is decreasing due to the use of anti-retroviral therapy (ART) and initiation of routine prophylaxis at low CD4 counts. ${ }^{10}$

In HIV-uninfected patients PCP is an emerging threat, and those who are at the highest risk for infection are transplant patients, those with cancer, chronic renal failure patients, patients with connective tissue diseases, and those who receive long courses of glucocorticoids, chemotherapy and other immunosuppressive agents. ${ }^{4.11,14,15}$

\section{Symptoms / presentation}

The clinical presentation of PCP is different in HIV-infected and HIV-uninfected patients. ${ }^{14,16}$ These differences are mainly due to a severe inflammatory response in HIV-uninfected patients, which is not possible in the HIV-infected population. ${ }^{14,17}$ The severity of PCP is therefore better determined by the inflammatory response of the host's body than by the load of organisms. In HIV-infected patients, colonisation of the lungs by $P$. jirovecii is very high, but the inflammatory response of the host is ineffective. ${ }^{2,14}$ As a result, the clinical presentation of PCP in this patient group is often insidious. The most common manifestations of PCP are gradually progressive dyspnoea, fever, non-productive cough, and chest discomfort that deteriorates within days to weeks before diagnosis. Patients typically present with these manifestations after two weeks to two months of the onset of symptoms. ${ }^{12,14}$ HIV-infected PCP patients have better oxygenation and survival rates, and this may be due to the smaller number of inflammatory cells in this group. ${ }^{2}$
Another factor that could contribute to better outcomes in this group is a higher index of suspicion for PCP in HIV-infected patients by doctors, leading to more prompt diagnosis and initiation of appropriate treatment. ${ }^{16}$

In HIV-uninfected patients there are smaller numbers of the organism in the lungs, and this makes the detection and definitive diagnosis of PCP more difficult. ${ }^{14}$ As a result of this delay in diagnosis, the duration between hospital admission and commencement of treatment for these patients is longer, thereby worsening the prognosis.16 The prognosis of HIV-uninfected patients with PCP is worse than that of HIV-infected patients, with mortality rates varying from $30 \%$ to $60 \% .^{14,16}$

\section{Examination/physical signs}

The primary manifestation of Pneumocystis jirovecii in HIV-uninfected patients is pneumonia. In this patient group PCP initially manifests with fever, dyspnoea and dry cough, and then progress within one week to severe hypoxia, rapid respiratory deterioration and respiratory failure that requires mechanical ventilation. Respiratory insufficiency in these patients is severe, and the course of the pneumonia is fulminant. ${ }^{3,14,18}$ Should HIV-uninfected patients present with extra-pulmonary symptoms and shock, an alternative diagnosis should be considered. 3

Even though the progression is slow, PCP can lead to respiratory failure, septic shock and death in HIV-infected patients. ${ }^{13}$ Survival rates in mechanically ventilated patients are low, ${ }^{17}$ and overall mortality rates are between 10 and 20 percent. ${ }^{2,14}$ The prognosis of HIV-infected patients with PCP has not improved much over the last 25 years, despite major advances in the treatment of HIV/ AIDS during this time. ${ }^{16}$

Lung auscultation in PCP patients is often unrevealing, but inspiratory crackles are the most common finding. However, patients may have signs of respiratory compromise, which include cyanosis, tachycardia and tachypnoea. PCP may present with unilateral or bilateral pneumothorax, which is manifested by pleuritic chest pain and dyspnoea. ${ }^{1,2,18}$

\section{Special investigations \\ Radiological investigations}

Chest radiography retains a key role in the clinical diagnosis of pneumonia in the immunocompromised, even though the X-ray can be normal at initial presentation. It is the prime modality in the diagnosis of pneumonia, and follow-up imaging is used to check for resolution. ${ }^{19}$ 
The diagnosis of PCP is suspected by the characteristic appearance of the chest X-ray, which shows bilateral or diffuse ground-glass opacities. In some patients with PCP the ground-glass opacities are distributed in the sub-pleural lung parenchyma, whereas peripheral sparing of ground-glass opacities occurs in other patients. ${ }^{14}$ Extensive areas of symmetrical, bilateral ground-glass opacities are not seen in other types of pneumonia. ${ }^{3}$

Appearances on radiographic imaging in HIV-infected and HIV-uninfected patients are similar, ${ }^{3,16}$ but may be varied, non-specific, and lag behind the symptoms. The classic appearance is of bilateral symmetrical peri-hilar or diffuse interstitial ground-glass opacification, as seen in Figure 1, but may be reticular or finely granular in appearance. If left untreated, this may progress to alveolar consolidation in three or four days. Infiltrates clear within two weeks, but in some patients infection will be followed by coarse reticular opacification and fibrosis. ${ }^{3,9,20}$

Although the findings are similar, ground-glass opacities may be more extensive and spread more rapidly, with-

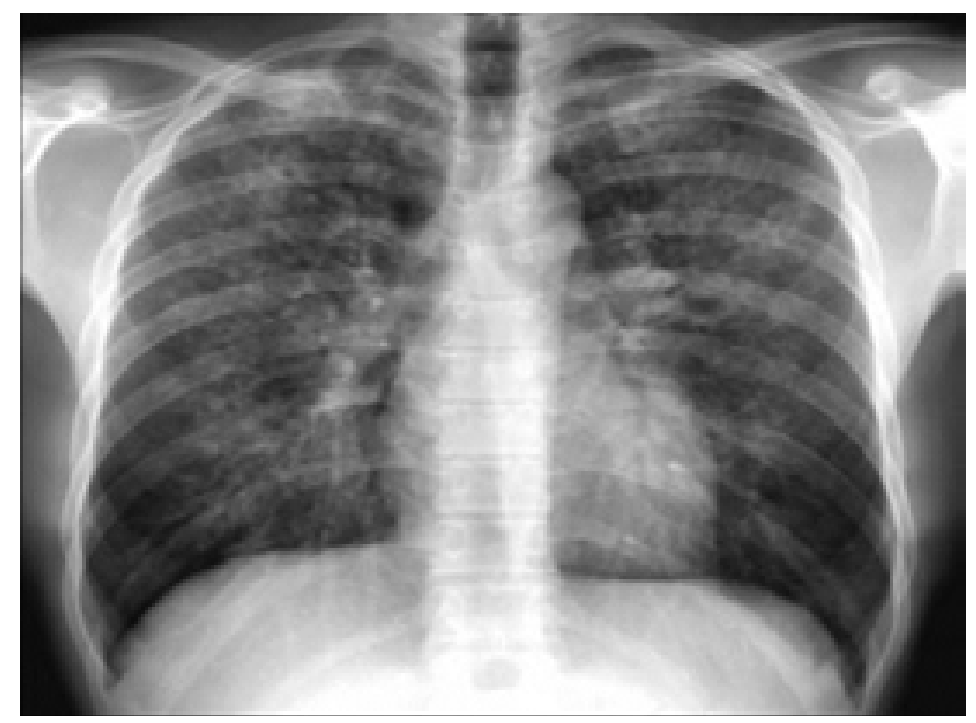

Figure 1: Typical chest X-ray of Pneumocystis jirovecii pneumonia in an HIV-infected patient. The X-ray shows ground-glass opacities, diffuse patchy infiltrates and pneumatocoeles (arrows). Source: Zhao JB. Pneumonia in immunocompromised patients. Medscape. 2017. Available at: https://emedicine.medscape.com/article/807846-oerview ${ }^{21}$

out peripheral sparing in HIV-uninfected patients. Lung consolidations may be more common in these patients due to pulmonary damage of the inflammatory immune response. ${ }^{3,18}$ Although radiographic imaging is not a definitive diagnostic tool, it is used clinically as PCR, serum $\beta$-D-glucan and microscopy which are more definitive are expensive and not always available in the African clinical context. ${ }^{4,14,16}$
In some instances PCP presents with normal chest radiographs. Chest high-resolution computed tomography (HRCT) may be useful in these patients, demonstrating patchy areas of ground-glass opacities, ${ }^{1,3,14}$ and with extensive ground-glass opacities being the main findings, as seen in Figure 2. A normal HRCT may in fact be used to exclude a diagnosis of PCP. 3,18 


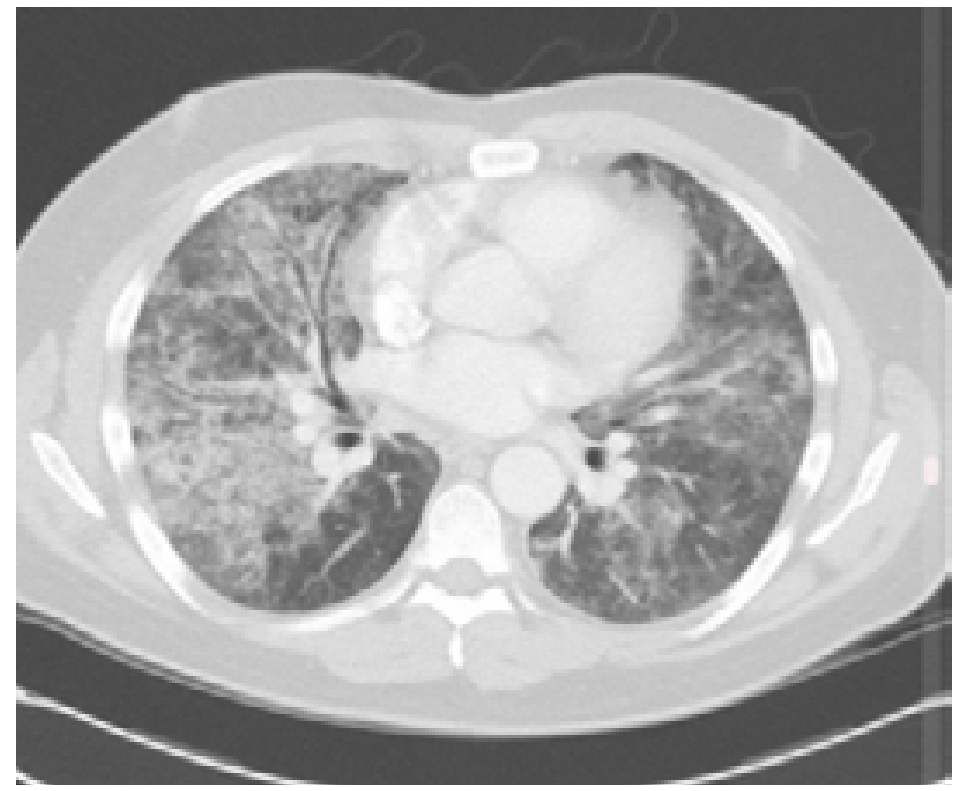

Figure 2: Diffuse ground-glass opacities, thickening of interlobular septa and linear opacities (arrows) in a chest CT-scan of a HIV-infected patient with PCP. Source: Zhao JB. Pneumonia in immunocompromised patients. Medscape. 2017. Available at: https://emedicine.medscape.com/article/807846-overview ${ }^{21}$

\section{Microscopy and culture}

Microscopic visualisation of Pneumocystis jirovecii is the gold standard for the diagnosis of PCP, because the organisms are very difficult to culture.18 Microscopy is done with organisms in sputum or broncho-alveolar lavage fluid.18 Sputum induction with hypertonic saline should be the initial procedure for microscopic diagnosis, especially in HIV-infected patients. ${ }^{2}$ However, HIV-uninfected patients have a lower number of the organisms, thereby making visualisation under microscopy more difficult, and rendering microscopic diagnosis with weak sensitivity for PCP. ${ }^{3,14}$ Giemsa staining is often used for microscopy, as seen in Figure 3, and the use of an immunofluorescence assay with anti-Pneumocystis antibodies can improve the microscopic visualisation of cysts. ${ }^{3}$

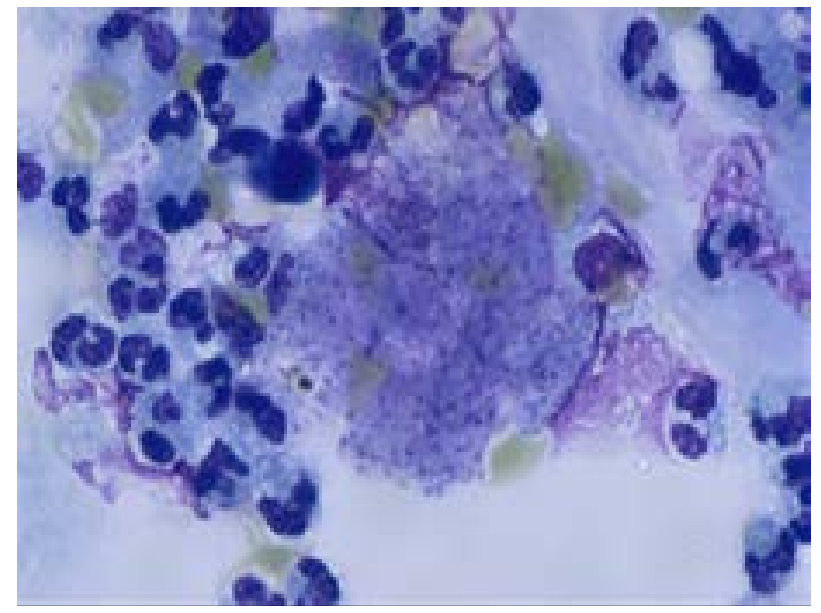

Figure 3: A cytological preparation from a broncho-alveolar lavage shows the faint bluish, dot-like intra-cystic bodies (arrow) of Pneumocystis jirovecii with Giemsa stain at high magnification. Source: AIDS Pathology. The Internet Pathology Laboratory for Medical Education, The University of Utah. 2017. Available at: https://ibrary.med.utah.edu/WebPath/TUTORIAL/AIDS/AIDS091.htm2 ${ }^{2}$ 


\section{Serum $\beta$-D-glucan}

Serum $\beta$-D-glucan (BDG) is derived from the cell wall of several fungi, including Pneumocystis species., and although it is not specific for Pneumocystis, measurement of serum BDG has been used for the diagnosis of PCP. ${ }^{14}$ The serum BDG test has a good negative predictive value, and the diagnostic performance of the test seems to be better for PCP than for invasive fungal infections like aspergillosis or candidiasis. ${ }^{3}$ Serum BDG is assayed from a blood sample, requiring a red-top bottle. These red top collection glass tubes are vacusera serum clot activator tubes as per recognized international standards. BDG may however also be assayed on bronchial washings. ${ }^{4} \mathrm{BDG}$ assays from broncho alveolar washings is often elevated in patients with PCP. The assay sensitivity appears to be high, and thus a diagnosis of PCP is less likely in patients with low levels (e.g. $<80 \mathrm{pg} / \mathrm{ml}$ using the Fungitell assay). The specificity for establishing a PCP diagnosis is low, since many other fungal diseases, as well as haemodialysis cellulose membranes and some drugs can produce elevation. ${ }^{4}$

\section{Polymerase chain reaction (PCR)}

PCR is used increasingly for the diagnosis of PCP due to its high sensitivity of $94 \%$ to $100 \%{ }^{14}$ This technique is often used in HIV-uninfected patients due to the high sensitivity.4,16 A negative PCR result allows for the withdrawal of PCP treatment, due to the high negative predictive value of PCR for PCP. ${ }^{14,16}$

\section{Bronchoscopy}

If the initial specimen of induced sputum (induced sputum by means of nebulization or physiotherapy where the patient does not have a productive cough) is negative for PCP then bronchoscopy with broncho-alveolar lavage (BAL) should be performed. ${ }^{2} \mathrm{HIV}$-uninfected patients often have rapidly progressive respiratory insufficiency, thereby making diagnosis through bronchoscopy difficult. ${ }^{14} \mathrm{BAL}$ fluid is however often required for microscopic identification of Pneumocystis jirovecii, ${ }^{18}$ as deep lung samples are required. ${ }^{4}$ BAL fluid has a $90 \%$ sensitivity for detecting PCP. Transbronchial biopsies are not commonly performed during bronchoscopy, because pneumothoraces are more common in PCP and can be iatrogenically caused by this procedure. ${ }^{4}$

\section{Management}

Cotrimoxazole (trimethoprim/sulfamethoxazole) is the treatment of choice for PCP of any severity in HIV-infected and HIV-uninfected patients. Treatment duration of 21 days is recommended for HIV-infected patients, and 14 days for HIV-uninfected immunocompromised patients. ${ }^{3,4,14,23}$ The longer duration of treatment for HIV-positive patients is due to the higher number of organisms, a delayed clinical response to treatment, and a high probability of relapse after a shorter treatment course. ${ }^{3,14}$ The treatment can be prolonged in HIV-uninfected patients if they show a poor clinical response to treatment, or if they are severely immunocompromised. ${ }^{11,14}$ Hospitalised patients should receive intravenous therapy until they improve enough to reliably absorb oral medication, whilst those with mild disease can be treated with oral therapy. HIV-positive patients who are not on ART should be initiated on it. ${ }^{1,12,23}$ Failure to respond clinically to cotrimoxazole cannot be decided on until one week of treatment has been completed. ${ }^{4}$

Cotrimoxazole is given in six-hourly doses for 21 days. The dosage is according to the patient's weight; trimethoprim is given at $15-20 \mathrm{mg} / \mathrm{kg}$, and sulfamethoxazole at 75-100 mg/ kg. ${ }^{3,23}$ Patients with a weight of less than $60 \mathrm{~kg}$ require three single-strength tablets, and patients with a weight of more than $60 \mathrm{~kg}$ will require four single-strength tablets. Treatment is given intravenously to patients who cannot swallow tablets for reasons such as excessive vomiting. ${ }^{12,23}$

Side-effects of cotrimoxazole include nephrotoxicity, hepatotoxicity, bone marrow depression, and skin rash, and these could be an obstacle to adherence to treatment and completion of the full treatment period. ${ }^{14,16}$ Patients who are allergic to cotrimoxazole should ideally be desensitised, as this is the most effective form of treatment. If there is a history of severe allergic reactions, e.g. Stevens-Johnson syndrome, cotrimoxazole should be avoided, and desensitisation should not be attempted. ${ }^{11}$

If a patient develops PCP despite being on prophylaxis, the treatment agent should be different from the prophylaxis agent if the clinician believes that the patient had been compliant to the prophylaxis. The different options for treatment are summarised in Table 1. 
Table 1: Treatment options for PCP. Source: Venkatesan P. Guidelines for the treatment of Pneumocystis jirovecii pneumonia (PCP) in adults. February 2017. Available from: www.nuh.nhs.uk/handlers.downloads.ashx?id=69347

\begin{tabular}{|c|c|c|c|}
\hline \multicolumn{2}{|c|}{ Severity of infection } & Drug options & \multirow{3}{*}{\begin{tabular}{l}
\multicolumn{1}{c}{ Adjunctive steroids } \\
Prednisone: \\
40 mg to be given $12-$ \\
hourly for five days. \\
(An equivalent dose of \\
an intravenous steroid \\
can also be given \\
initially.)
\end{tabular}} \\
\hline First-line: & $\begin{array}{l}\text { For all degrees } \\
\text { of severity }\end{array}$ & Cotrimoxazole & \\
\hline \multirow[t]{3}{*}{$\begin{array}{l}\text { Second- } \\
\text { line: }\end{array}$} & $\begin{array}{l}\text { Mild PCP } \\
\text { infection }\end{array}$ & $\begin{array}{ll}\text { In descending } & \text { order of } \\
\text { preference: } & \\
\text { Dapsone } & \text { and } \\
\text { trimethoprim } & \\
\text { Atovaquone } & \end{array}$ & \\
\hline & $\begin{array}{l}\text { Moderate PCP } \\
\text { infection }\end{array}$ & $\begin{array}{l}\text { In descending order of } \\
\text { preference: } \\
\text { Clindamycin and } \\
\text { primaquine } \\
\text { Dapsone and } \\
\text { trimethoprim }\end{array}$ & \multirow{2}{*}{$\begin{array}{l}40 \mathrm{mg} \text { to be given in the } \\
\text { morning. Continue for } \\
\text { the next five days. } \\
20 \mathrm{mg} \text { to be given in the } \\
\text { morning, continue for } \\
\text { the next eleven days. } \\
\text { Steroids should be } \\
\text { discontinued with or } \\
\text { before acute anti-PCP } \\
\text { treatment }\end{array}$} \\
\hline & $\begin{array}{l}\text { Severe PCP } \\
\text { infection }\end{array}$ & $\begin{array}{l}\text { In descending order of } \\
\text { preference: } \\
\text { Clindamycin and } \\
\text { primaquine } \\
\text { Pentamidine IV }\end{array}$ & \\
\hline
\end{tabular}

\section{Clindamycin and primaquine Pentamidine IV}

Oxygen by facemask or continuous positive airway pressure (CPAP) should be given to hypoxic patients as necessary.

Oral prednisone should be added for 21 days. The adjunctive steroid guideline is summarised in Table 1. The use of corticosteroids in conjunction with anti-Pneumocystis therapy decreases the incidence of mortality and respiratory failure associated with severe PCP when administered in the first 72 hours of treatment, but should preferably be initiated with the anti-PCP treatment. ${ }^{4,12,18,23}$ Corticosteroids blunt the inflammatory response in the patient's lungs that was initiated by the degradation and clearance of organisms, thereby attenuating lung injury. ${ }^{14}$ Patients on anti-PCP treatment should be monitored regularly, and oxygen saturation, pulse rate, blood pressure and temperature should be recorded. If the condition of the patient deteriorates consider the development of a pneumothorax, an additional infection, progression of PCP despite treatment, or treatment toxicity. ${ }^{4}$

\section{Prophylaxis}

Mortality of PCP remains high despite intensive treatment, and therefore prophylaxis is justified. ${ }^{14}$

Drug prophylaxis reduces the incidence of PCP and lengthens the disease-free intervals between episodes. There is currently no PCP vaccine available.

Prophylaxis should be considered for: , $^{2,4,11,12,23}$

- All patients with a history of Pneumocystis infec-

tion

- $\quad$ Severely immunocompromised patients

- $\quad$ All HIV-positive individuals with a CD4+ T-cell count below 200 cells $/ \mathrm{mm} 3$

- $\quad$ Patients with malignancies

- Organ transplant recipients

- Patients with vasculitis and inflammatory diseases

- Patients with inflammatory bowel disease.

Prophylaxis should continue until immunity recovers sufficiently. Oral cotrimoxazole is the drug of choice in HIV-infected and HIV-uninfected patients. ${ }^{12,14,23}$ The prophylactic dosage is two single strength tablets daily. ${ }^{23}$ An alternative regimen is cotrimoxazole (80/400) one tablet daily, or two tablets three times per week. ${ }^{14}$ 
Dapsone, pentamidine and atovaquone have also been used for prophylaxis as second-line in patients who cannot tolerate cotrimoxazole. ${ }^{2,3,12}$ The recommended dose for dapsone is $2 \mathrm{mg} / \mathrm{kg} /$ day or $4 \mathrm{mg} / \mathrm{kg} /$ week. The maximum daily dose is $100 \mathrm{mg}$.12 When prescribing dapsone ensure that the patient does not have G6PD deficiency. ${ }^{12}$ Patients (particularly children) who start ART, attain low $\mathrm{HIV}$ viral loads and improved CD4 counts of $>200$ cells $/ \mathrm{mm} 3$ for three consecutive months, may discontinue prophylactic therapy safely. ${ }^{12,23}$

\section{Secondary prophylaxis}

Patients who had PCP infection, who have been treated and are without symptoms, receive secondary prophylaxis or chronic maintenance therapy, which conforms to the same schedule as primary prophylaxis for each patient group. ${ }^{12,23}$ In descending order of preference the options for secondary prophylaxis are cotrimoxazole, dapsone, atovaquone and aerosolised pentamidine. ${ }^{4}$

Secondary prophylaxis should be started concurrently with the end of PCP treatment and continued for life, unless immune reconstitution occurs with ART. ${ }^{12,23}$

Secondary prophylaxis is discontinued when patients have immune reconstitution and a CD 4 count increased from $<200$ cells $/ \mathrm{mm} 3$ to $>200$ cells $/ \mathrm{mm} 3$ for three consecutive months as a result of ART. Prophylaxis should be continued for life in patients who develop PCP despite immune reconstitution. ${ }^{12,23}$

\section{Special considerations during pregnancy}

Pneumonia, including PCP, can cause acute lung injury in pregnant women. Although PCP can have an insidious onset, it can progress rapidly to acute respiratory failure and death for both the mother and the foetus. ${ }^{24}$

Prevention is the best form of PCP management in pregnancy. Cotrimoxazole is the agent of choice for chemoprophylaxis in pregnant patients with immunosuppression, ${ }^{12,24}$ is safe in pregnant and breastfeeding HIV-positive patients, and risks to the foetus are outweighed by the benefits. ${ }^{24}$

PCP in pregnancy has an aggressive course, and maternal and foetal outcomes are generally poor. PCP in pregnancy should therefore be managed by a multidisciplinary team that involves a physician, obstetrician and pulmonologist, and includes high doses of cotrimoxazole and oxygen therapy. ${ }^{12,24}$

Acutely ill pregnant patients present an ethical dilemma to the treating team. Delivery of the foetus may improve maternal ventilation and outcomes, but could compromise the foetus, with complications like stillbirth and congenital transplacental infection. ${ }^{24}$

\section{Key messages}

- $\quad$ PCP is a common cause of morbidity and mortality in all immunocompromised patients.

- The classical presentation is initial fever, dyspnoea and cough.

- In HIV-uninfected patients the course of the disease is fulminant and rapid with high mortality rates.

- In HIV-infected patients the course of the disease is insidious, but can however lead to shock, respiratory failure and death.

- $\quad$ Radiographic imaging shows ground-glass opacities.

- The drug of choice for treatment and chemoprophylaxis is cotrimoxazole.

\section{Conflict of interest disclosure}

All the authors declare that they have no conflicts of interests.

\section{References}

1. Huang L, Cattamanchi A, Davis L, Den Boom S, Kovacs J et al. HIV-Associated Pneumocystis Pneumonia. Proc Am Thorac Soc. 2011; 8: 294-300 PubMed . DOI 10.1513/pats.201009-062WR

2. Thomas CF, Limper AH. Pneumocystis Pneumonia. NEJM. 2004; 30(24): 2487 PubMed -97. DOI 10.1056/ NEJMra032588

3. Roux A, Gonzalez F, Roux M, Mehrad M, Menotti J, et al. Update on Pneumocystis jirovecii infection in nonHIV patients. Medicine et maladies infectieuses. 2014; 44: 185198. DOI 10.1016/j.medmal.2014.01.007

4. Venkatesan P. Guidelines for the treatment of Pneumocystis jirovecii pneumonia (PCP) in adults. February 2017. Available from: www.nuh.nhs.uk/handlers. download.cfm $/$ doc $=$ docm93jij,42647.pdf\&ver $=4793$ Accessed on 1 November 2017.

5. Readhead SA, Cushion MT, Frenkel JK, Stringer JR. Pneumocystis and Trypanossoma cruzi: nomenclature and typifications. J Eukaryot Microbiol 2006; 53(1):2-11. DOI 10.1111/j.1550-7408.2005.00072.x

6. Stringer JR, Beard CB, Miller RF, Wakefield AE. A new name for Pneumocystis from humans and new perspectives on the host-pathogen relationship. Emerg Infect Dis. 2002; 8(9): 891-896. DOI 10.3201/eid0809.020096 
7. Minielly JA, Holley KE. Pneumocystis carinii Pneumonia. Canad Med Ass J. 1969. 100: 846-854. PMID 5305582 PMC 1945203

8. Morris A, Lundgren JD, Masur H, et al. Current epidemiology of Pneumocystis pneumonia. Emerg Infect. Dis. 2004; 10 (10): 1713-1720. DOI 10.3201/eid1010.030985 9. Allen CM, AL-Jahdali HH, Irion KL, Al Ghanem S, Gouda A, Khan AN. Imaging lung manifestations of HIV/AIDS. Thorac Med. 2010; 5(4): 201-216. DOI 10.4103/1817-1737.69106

10. Guo F, Chen Y, Yang SL, Xia H, Li XW, et al. Pneumocystis Pneumonia in HIV-Infected and Immunocompromised Non-HIV infected Patients: A Retrospective Study of two centers in China. PLoS One. 2014. 9(7): e101943. DOI 10.1371/journal.pone.0101943

11. Wei KC, Sy C, Wu SY, Chuang TJ, Huang WC, Lai PC. Pneumocystis jirovecii pneumonia in HIV-uninfected, rituximab treated non-Hodgkin lymphoma patients. Sci Rep. 2018;8(1):8321. Published 2018 May 29. doi:10.1038/ s41598-018-26743-4

12. Guidelines for Prevention and Treatment of Opportunistic Infections in HIV-Infected Adults and Adolescents. AIDS info. 2013. Available at http:/ / aidsinfo.nih. gov/contentfiles/lvguidelines/adult_oi.pdf Accessed 13 November 2017.

13. Wang H, Lin C, Kuo C, Liu C, Lee C. Mortality predictors of Pneumocystis jirovecii pneumonia in human immunodeficiency virus-infected patients at presentation: Experience in a tertiary care hospital in northern Taiwan. J Microbiol Immunol Infect. 2011; 44: 274-281 PubMed . DOI 10.1016/j.jmii.2010.08.06

14. Tasaka S, Tokuda H. Pneumocystis jirovecii pneumonia in non-HIV-infected patients in the era of novel immunosuppressive therapies. I Infect Chemother. 2012; 18: 793-806 PubMed . DOI 10.1007/s10156-012-0453-0

15. Carmona EM, Limper AH. Update on the Diagnosis and Treatment of Pneumocystis Pneumonia. Therapentic Advances in Respiratory Disease. 2011, 41-59. DOI:10.1177/1753465810380102.
16. Li M, Lee N, Lee C, Lee H, Chang C, Ko W. Pneumocystis jirovecii pneumonia in immunocompromised patients: Delayed diagnosis and poor outcomes in non-HIV-infected individuals. J Microbiol Immunol Infect. 2014; 47: 42-47. DOI 10.1016/j.jmii.2012.08.024

17. Kotani T, Katayama S, Miyazaki Y, Fukuda S, Sato Y, Ohsugi K. Risk factors for the mortality of Pneumocystis jirovecii pneumonia in non-HIV patients who required mechanical ventilation: a retrospective case study series. BioMed Research International. 2017; Article ID 7452604. DOI 10.1155/2017/7542604

18. Kanne JP, Yandow DR, Meyer CA. Pneumocystis jirovecii pneumonia: High-Resolution CT findings in patients with and without HIV infection. Cardiopulmonary Imaging. 2012; 198: 555-561. DOI 10,2214/AJR.11.7329

19. Reynolds JH, Banerjee AK. Imaging pneumonia in immunocompetent and immunocompromised individuals. Curr Opin Pulm Med. 2012; 18(3):194 PubMed -201. DOI 10.1097/MCP.0b013e328351f953

20. Boiselle PM, Crans CA, Jr, Kaplan MA. The changing face of Pneumocystis carinii pneumonia in AIDS patients. AJR Am J Roentgenol. 1999; 172:1301-1309 PubMed . DOI 10.2214/ajr.172.5.10227507

21. Zhao JB. Pneumonia in immunocompromised patients. Medscape. 2017. Available at: https://emedicine.medscape.com/article/807846-overview Accessed 13 November 2017.

22. AIDS Pathology. The Internet Pathology Laboratory for Medical Education, The University of Utah. 2017. Available at: https://library.med.utah.edu/WebPath/TUTORIAL/AIDS/AIDS091.html Accessed 11 November 2017. PMCID PMC35228

23. Meintjes G, Moorhouse MA, Carmona S, Davies $\mathrm{N}$, Dlamini S, et al. Adult anti-retroviral therapy guidelines 2017. S Afr J HIV Med. 2017;18(1), a776. DOI 10.4102/ sajhivmed.v18i1.776

24. Ngwenya S. Pneumocystis carinii Pneumonia; Lost lives in pregnancy: chemoprophylaxis saves lives. Pulm Res RespirMed Open J. 2016; 3(3): 41-44. DOI 10.17140/PRRMOJ-3-131 